\title{
Osteogenic Effects of IL-12 on Bone Marrow Mesenchymal Stem Cells Facilitates Its Irradiation Hematopoiesis Recovery
}

Fengjie Li

Xinqiao Hospital

Rong Zhang

Xinqiao Hospital

Changpeng $\mathrm{Hu}$

Xinqiao Hospital

Qian Ran

Xinqiao Hospital

Li Chen

Xinqiao Hospital

Yang Xiang

Xinqiao Hospital

Lixin Xiang

Xinqiao Hospital

Yang Yang

Xinqiao Hospital

Gang Zhang

Xinqiao Hospital

Zhongjun Li ( $\square$ Zhongjunli@tmmu.edu.cn )

https://orcid.org/0000-0001-7629-0911

\section{Research}

Keywords: IL-12, bone marrow mesenchymal stem cells, osteogenesis, irradiation, hematopoiesis

Posted Date: January 14th, 2020

DOI: https://doi.org/10.21203/rs.2.20812/v1

License: (1) (1) This work is licensed under a Creative Commons Attribution 4.0 International License.

Read Full License 


\section{Abstract}

\section{Background}

Impaired osteogenesis differentiation of bone marrow mesenchymal stem cells (BM-MSCs) results inefficient physical supporting of hematopoietic niche and further counts against hematopoiesis recovery in radiotherapy. Interleukin-12 (IL-12) holds facilitations on acute irradiation hematopoietic recovery, but the mechanisms remain to be clarifiedAim To investigated the osteogenic effects of IL-12 on BM-MSCs and the relationship between IL-12's osteogenic effects of BM-MSCs and its hematopoietic supportive role.

\section{Methold}

The osteogenic effects of IL-12 in vivo were investigated by estimating the osteoblasts in femurs of Balb/c mice after 5 Gy 60 Co-y irradiation, and the osteogenic effects of IL-12 in vitro were investigated by analyzing the osteogentic genes and calcium mineral deposits formations in BM-MSCs after 9 Gy 60 Co-yirradaition at different times of co-culturing with IL-12 in osteogenic induction medium. For the latter, the special siRNA of IL-12 receptor 1 (IL-12Rß1), tyrosine kinase 2 (TYK2) and the inhibitor of signal transducer and activator of transcription3 (STAT3) were used to explore the possible mechanism. The bone marrow hematopoietic supportive role of cells co-cultured with IL-12 were also investigated by analyzing the clone-forming of hematopoietic progenitor/ stem cells (HSPCs).

Results

Interleukin-12 (IL-12) holds facilitaties on acute irradiation-induced hematopoietic and osteogenesis damages in Balb/c mice. IL-12 also promoted osteogenesis differentiation of irradiated BM-MSCs, and for that, especially during early period after irradiation, the IL-12 receptor 1 (IL-12Rß1)/ tyrosine kinase 2 (TYK2)/ signal transducer and activator of transcription3 (STAT3) signaling played a crucial role. That was reflected by obvious synchronicity between the upregulation time of IL-12Rß1 and the time of the better IL-12 induced osteogenesis of BM-MSCs. The more osteogenic genes expressions and more calcium mineral deposits formations in BM-MSCs were obtained in cells co-cultured with IL-12 within 1 hour after irradiation; Beside that IL-12 increased the phosphotyrosine of STAT 3 ( $p$-STAT3) which along with IL-12 induced osteogenesis promotions were abrogated by using IL-12Rß1 and Tyk2 silencing RNA (siRNA) or inhibitor of STAT3. Further, the clone-forming of hematopoietic progenitor/ stem cells (HSPCs) showed much better in IL-12-BM-MSCs transplanted femurs of irradiated mice than that of BM-MSCs, even cells were co-cultured with IL-12 for only 24 hours.

\section{Conclusion}

IL-12 may assist hematopoiesis recovery through its early osteogenic differentiation promotion of BMMSCs after irradiation, which suggested potential therapeutic targets of bone marrow hematopoietic damages after radiotherapy. 


\section{Background}

Radiotherapy is considered to be one of most accepted medical and widely used treatments for tumor and cancer. However, even the lowest dose of localized irradiation exposure can result in adverse complications to adjacent organs, tissues, especially irradiation-sensitive organizations, such as hematopoietic and gastrointestinal system, gonads tissues[1]. The irradiation caused myelosuppression and hematopoietic dysfunction [1, 2] have become common side effects and obstacles in radiotherapy patients[3-7]. As we known, hematopoiesis is a complex and multistep developmental process involves pluripotent hematopoietic stem and progenitor cells (HSPCs), varies supportive cells in the hemopoietic microenvironment (HM) and terminating erythrocytes. In irradiation, HSPCs were seriously damaged but for more importance the hemopoietic microenvironment (HM) which regulates the hematopoietic process, including maintenances and regulation of HSPCs quiescence, self-renewal and differentiation were also damaged. Then the demaged HM resulted its ineffective supporting in hematopoiesis recovery[1]. Preserving and promoting $\mathrm{HM}$ physiological function gradually become the target of hematopoietic reconstitution and rescuing hematopotic injury [6-8] .

BM-MSCs, a population of adult stem cells, have considerable differentiation capacity into multi-lineages cells/tissues. For the bone marrow hematopoiesis, BM-MSCs are important components and regulators of the participating in HM formation $[4,5,9]$. And for more importance, BM-MSCs are the main source of formation of osteoblasts [10], which constitute the physical support of of HM directly or indirectly regulating its size and activity and offering hematopoiesis supporting functions [10-12]. In irradiation, BM-MSCs also have more redioresistance than HSPCs[3, 13], but their osteoblasts differentiation potential were obviously decreased, which subsequent crippled their hematopoiesis-supporting $[2,13,14]$.

IL-12, produced by monocytes, macrophages and dendritic cells, has various effects, including anticancer, anti-infection and curing of autoimmune diseases[15-17]. Recent evidences have indicated its favourable mitigation acute irradiation injury effects, including remarkable hematopoiesis recovery [18] and damaged organs recovery effects [19-23]. Although these studies have indicated that IL-12 may protect or facilitate hemato poiesis by directly and/or indirectly interacting with HSPCs [18, 24, 25], mechanisms of how it acts on HSPCs and whether it acts on the HM to assist hematopoiesis recovery remain to be clarified.

In present study, we focus on osteogenesis effects of IL-12 on BM-MSCs and its possible mechanisms and its relationship with irradiation hematopoiesis recovery. We first found single dose of IL-12 have a remarkable osteogenesis promotion in vivo and vitro. During the early period of IL-12 osteogenic differentiation promotion in irradiated BM-MSCs, the IL-12RB1/TYK2/STAT3 signal plays an indispensable role. Beside that, we also found, BM-MSCs co-cultured with IL-12 for 24 hours had significant more bone formation and hematopoiesis supporting functions. These findings furthered our understanding of hematopoiesis recovery effects of IL-12 in irradiation and provided potential target of rescuing differentiation bias of BM-MSCs to promote the hematopoietic supporting in radiotherapy. 


\section{Methods}

Animals

Male and female Balb/c mice, aged 4-6 weeks, were provided by the Experimental Animal Center of The Third Military Medical University and housed in a specific pathogen-free animal facility of Laboratory Animal Centre in the Second Affiliated Hospital of Army Medical University. All experiments in this study were approved by the Animal Experiment Ethics Committee of Army Medical University institute

Cell culture

$\mathrm{Balb} / \mathrm{c}$ mice BM-MSCs, complete medium containing and osteogenesis inducing medium were purchased from Cyagen Biosciences Inc. Guangzhou, China. Cells were cultured at $37^{\circ} \mathrm{C}$ under $5 \% \mathrm{CO}_{2}$ atmosphere. The culture medium was replaced every 2 to 3 days. Cells at passage 11 (P8) were used in subsequent experiments.

Pre-conditioning irradiation

For all irradiation procedures, both cells and animals were total body irradiated (TBI) with a single dose of ${ }^{60} \mathrm{Co}-\mathrm{y}$ using an irradiation source[3] with a source-surface distance of $150 \mathrm{~cm}$ and at a rate of $0.69 \mathrm{~Gy} / \mathrm{min}$ at the Irradiation Research Centre of the Third Military Medical University. The dose for cells was $9 \mathrm{~Gy}$ and for animals was $5 \mathrm{~Gy}$.

Reagents

The mouse recombinant IL-12 was purchased from Biolegend, San Diego, CA, USA. Small interfering RNA (siRNA) targeting mouse Tyk2 was chemically synthetic in Ribo Bio Co. Ltd., Guangzhou, China. Small interfering RNA targeting mouse IL-12R 31 were purchased from Santa Cruz Biotechnology Co. The inhibitor of STAT3, cryptotanshinone (CPT), was purchased from Med Chem Express. The bone marrow wash medium, StemSpan ${ }^{\text {TM }}$ SFEM culture medium, the methylcellulose-based medium and the culture plates, SmartDish ${ }^{\text {TM }}$ plate were purchased from Stemcell Technologies, Canada.

The primary antibodies used for western blotting were following: anti-IL-12Rß1 (Bioss), anti-IL-12Rß2 (Bioss), anti-RUNX2 (Runt-related transcription factor 2, Santa Cruz Biotechnology), anti-Osteoglycin (OGN, Santa Cruz Biotechnology), anti- $\beta$-actin (Santa Cruz Biotechnology), anti-phosphorylated STAT3 (Tyr705) mouse monoclonal (mAb) (Cell Signaling Technology), anti-STAT3 (124H6) mouse mAb (Cell Signaling Technology), rabbit anti-TYK2 antibody (Abnova, Taipei, Taiwan).

Quantitative polymerase chain reaction

Total RNA was isolated using Trizol reagent (Invitrogen. China) and the first-strand cDNA was synthesized using a reverse transcription reagent kit (TOYOBO, FSK-100, Osaka, Japan) accordance with the manufacturer's instructions. Next, real-time qPCR (RT-qPCR) was performed in triplicate using Fast 
Start Universal SYBR Green Master Mix (Roche, Germany). The data were analysed using the delta-delta Ct method. Experiments were performed in triplicate.

The sequences of the primers used in RT-qPCR are as follows: GAPDH forward, 5'GACATCAAGAAGGTGGTGAAGC-3'; GAPDH reverse, 5'-GAAGCTGGAAGAGTGGGAGTT-3'; Runx2 forward, 5'-GTGCCCAGGCGTATTTCAGATG-3'; Runx2 reverse, 5'-GCGGGGTGTAGGTAAAGGTCGC-3'; Ogn forward, 5'-GTGGTCACATGGATAGCCTTTAGTC-3'; Ogn reverse, 5'-GAGCATATTTAGTTTGTTTGGGTGA-3'; IL-12Rß1 forward, 5'ATGCGCTGGTGGTCGAGATGC-3'; IL-12Rß1 reverse, 5'CCCGGCTCCGCAGTCTTATG-3'; IL-12Rß2 forward, 5'CGACGCTCTCAAAACTCACATCC-3'; IL-12Rß2 reverse, 5'TTTGCCGGAAGTAACGAATTGAG-3'; Tyk2 forward, 5'-GTGCCTTCCGTGTTCAGCGTGTG-3'; Tyk2 reverse 5'-GCCCAGAACGAATAGACTCAGGAA3 .

Western blotting

Total protein lysates were extracted with cell lysis buffer (Beyotime, Shanghai, China) and were denatured by boiling. Next, the cell lysates were separated on $4-12 \%$ SDS-polyacrylamide gels and transferred onto polyvinylidene fluoride membranes (PVDF Western Blotting Membranes, Roche). The membranes were blocked in PBS buffer ( $50 \mathrm{mM}$ Tris- $\mathrm{HCl}, 150 \mathrm{mM} \mathrm{NaCl}$, and $0.1 \%$ Tween [ $\mathrm{pH} 7.6$ ]) supplemented with $5 \%$ non-fat dry milk and were incubated with the appropriate antibodies for 12 hours. An HRP-labelled secondary antibody and a chemiluminescent detection system (Phototope-HRP western blot detection kit; New England Biolabs, Ipswich, MA) were used for developing the blots.

Hematopoiesis and osteobalsts detections

The peripheral blood cell counts (PBCC) were estimatied by analyzing the eyeball blood with the automatic animal blood cell analyzer (Prandre XFA6030, Nanjing Prande Medical Equipment Co., Ltd., China). For the hematopoiesis of bone marrow, the bilatteral femur were removed and fixed in $10 \%$ formalin and embedded in paraffin wax for micro-sectioning at $5 \mu \mathrm{m}$ and routine hematoxylin \& eosin $(\mathrm{HE})$ staining. All slides were examined under microscope (Axio Imager 2, Zeiss, Germany) and the volume of bone marrow hematopoietic tissues were detected by image analysis and reporting system (YC.YX-2050, Chongqing telemedicine equipment Co., Ltd. China.).

For bone marrow HSPCs colony assays, mice were euthanized by injecting $150 \mathrm{mg} / \mathrm{kg}$ sodium pentobarbital and their bilatteral femur were removed. The bone marrows were aseptically flushed into serum free expansion medium (StemSpan ${ }^{\text {TM }}$ SFEM, StemCell Technologies Inc, Vancouver, BC, Canada) using a syringe fitted with a $2 \mathrm{l}$-gauge needle. Cells were counted and cultured in methylcellulose media (MethoCult GF, StemCell Technologies Inc, Vancouver, BC, Canada) following the manufacturer's instruction. A total of 300000 cells from unirradiated mice were used as a normal control (Normal). The colony-forming units (CFU) of granulocyte (CFU-G) and macrophage (CFU-M), CFU-granulocyte macrophage (CFU-GM), CFU-granulocyte, erythroid, macrophage, megakaryocyte (CFU-GEMM) were analyzed according to the technical manual, mouse CFU assays using methoCult ${ }^{\mathrm{TM}}$ (Stemcell Technologies, Canada) in situ by light microscope (Leica DMIRB, Germany). 
BM-MSCs committed osteogenic differentiation detection were analyzed by mineralized matrix staining for calcium mineral deposits using alizarin red S staining kit (Cyagen, China). ALP activities was tested using ALP staining complied with 5-bromo-4-chloro-3-indolyl phosphate and nitro-blue-tetrazolium (BCIP/NBT) alkaline phosphatise colour development kit (Beyotime Biotechnology, Shanghai, China). The mineralized matrix were examined and photographed using a light microscope (Leica DMIRB, Germany). The bone mineral densities were analyzed at the bottom of the distal growth plate, where the epiphyseal cap structure completely disappeared and continued for 95 slices $(10.5 \mu \mathrm{m} /$ slice, using Scanco Viva CT40) towards the proximal end of the femur.

Cells transplantation assays

Before transplantations, irradiated mice were anesthetized with $20 \mu \mathrm{g} / \mathrm{kg}$ pentobarbital sodium, using a $10 \mu \mathrm{l}$ microliter syringe purchased from Gaoge indsstrial and trading co. LTD., and subsequently cells suspended in a minimal volume of $3 \propto$ I PBS and 10000 cells were injected intrafemorally through the patellar surface. The whole cells transplantaions were carried out within 1 hour after TBI.

Transfection, inhibition and immunofluorescence assays

Briefly, cells transfected with special siRNA (final concentration, $50 \mathrm{nM}$ ) by using Lipofectamine 3000 (Invitrogen, Carlsbad, CA, USA) following the manufacturer's instructions. After 72 hours of transfection, cells were co-cultured with or without $0.2 \mathrm{ng} / \mathrm{ml} \mathrm{IL-12} \mathrm{for} 24$ hours. For the inhibition of STAT3, the inhibitor were added in the culture medium at $50 \mathrm{nM}$ for co-culturing 24 hours, and then cells were co-

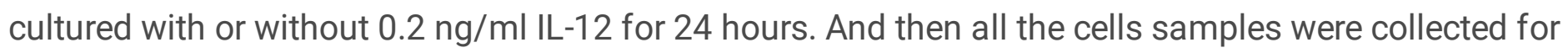
further detections.

Cells for immunofluorescence test were fixed with $4 \%$ formaldehyde and then were incubated overnight at $4{ }^{\circ} \mathrm{C}$ with a primary antibody and were incubated with FITC-labelled secondary antibody (Zhongshan Golden Bridge, China) for $1 \mathrm{~h}$ at RT. After each step, the prepared specimens were counterstained with $5 \mu \mathrm{g} / \mathrm{ml}$ 4,6-diamidino-2-phenylindole and were observed with a laser scanning confocal microscopy (LSCM) (Olympus, Japan).

Statistical analysis

All data were presented as the mean \pm SEM. Statistical analysis was performed using GraphPad Prism version 6.0. An independent unpaired $t$ test was used to compare data obtained from the experimental groups with those obtained from the control group. The results were considered significant at $* P<0.05$, $\star * P<0.01$, and $* * * P<0.001$.

\section{Results}

IL-12 promotes the hematopoiesis recovery 
To test the hematopoiesis effects of IL-12, we explored the bone marrow pathological, HSCPs colony formations and PBCC profiles in irradiated mice which were then promptly cured with a single dose of $1 \mu \mathrm{g} / \mathrm{Kg} \mathrm{IL}-12$ within 1 hour after irradiation through subcutaneously injection. As shown in Fig. $1 \mathrm{a}$, the structure and the cellularity of femurs from irradiated mice, both cured with or without IL-12, were severely damaged at the day 14 after irradiation. But IL-12 cured mice showed significant more hematopoietic tissues in femurs. (Fig. 1b).

The PBCCs in all irradiated mice took on a firstly decreasing and then recovery increasing change. But the IL-12 cured mice performed better profiles in blood cell change process. During the first declining phase, in IL-12 cured mice, PBCCs decreasing attenuated significantly, and during the latter recovery phase, nearly around day 14 after irradiation, PBCCs increasing accelerated significantly (Fig. 1c). The HSCPs colony formations, detected at the day 3 after irradiation, were also decreased in all irradiated mice, but in IL-12 cured mice, significant more colonies of CFU-G, CFU-M, CFU-GM and total CFU were reproduced (Fig. 1d, supplementary Fig. 1).

\section{IL-12 promotes the osteogenesis in vivo and in vitro}

We next examined of the effect of IL-12 on osteogenesis in vivo. Results showed osteoblasts around the endosteum of cancellous femurs of IL-12 cured mice were obvious more than that of without IL-12 cured ones (Fig. 2a). In vitro, the committed osteogenic differentiation of irradiated BM-MSCs co-cultured with IL-12 were also significantly increased. Beside that, IL-12 induced osteogenesis enhancing showed effective concentrations of $0.2-1 \mathrm{ng} / \mathrm{ml}$ (Fig. $2 \mathrm{~b}, \mathrm{c}$ ). These results indicated a apparent promotion of the osteogenesis after irradiation.

IL-12Rß1 plays a crucial signal in IL-12 induced early osteogenesis of BM-MSCs after irradiation

Considering IL-12 biological activities depended its receptors, IL-12R 31 and IL-12R 32 , they and their corresponding JAK/STATs were estimated in irradiational conditions. After irradiation, both IL-12Rß1, IL$12 R \beta 2$ expressions exhibited evident raising first and decreasing then. It is worth noting that IL-12R $\beta 1$ expression was more swift than that of IL-12R 32 , which was mainly manifested in the fact that the RNA expression peak of IL-12R $\beta$ appeared at 12 hours after irradiation, and the protein expression peak appeared at 24 hours after irradiation. while the protein expression peaks of IL-12Rß2 appeared at 24 hours and 72 hours after irradiation, respectively (Fig. 3a-c).

Based on the expression profiles of the IL-12R 1 and IL-12R 2 after irradiation, we tried to figure out whether they were correlated to IL-12-induced osteogenesis. We designed two experiments. First, irradiated cells were co-cultured with IL-12 for the whole experiment period, and for this, IL-12 were administrated within 1 hour after irradiation. The osteogenic genes were estimated at day 1 and day 3 after co-culturing with IL-12. Second, we investigated administration time effects of IL-12 on osteogenesis by analyzing the osteoblastic differentiation of irradiated cells co-cultured with IL-12 within 1 hour and at day 3 after irradiation. According to results, during first 3 days of co-culturing, the IL-12 was administrated within one hour after irradiation, expressions of Runx2 and Ogn were significantly 
increased, but the increasing expressions were more obvious in cells on day 1 (Fig. 3d-g). Beside that, IL12 administrated within 1 hour after irradiation induced more matrix mineralization in BM-MSCs than that IL-12 administrated on day 3 (Fig. 3g), suggesting that the earlier administration of IL-12 the better osteogenesis would be brought on, and it seems that IL-12 played a important role in the early stage of the osteogenesis process in BM-MSCs. These results not only confirmed IL-12-induced osteogenic enhancements of BM-MSCs, but also suggested a potential role of IL-12Rß1 in IL-12 induced osteogenic differentiation in irradiation.

The necessity of IL-12Rß1/TYK2/STAT3 signaling in IL-12-induced osteogenesis

To further elucidate the IL-12Rß1 signaling function in IL-12-induced early osteogenesis, the main downstream molecules of IL-12Rß1, TYK2 and STAT3, were evaluated. For this, the cells transfected with siRNA for 72 hours were co-cultured with IL-12 for 24 hours. Results showed that p-STAT3 activities and Runx2 and Ogn expressions were obvious promoted in IL-12 treated cells, and that were obviously abrogated by the special siRNA (Fig. 4).

Next, we used a special inhibitior of STAT3, CPT, specially inhibits the activation of STAT3 by selectively blocking the STAT3 Tyr705 phosphorylation and the dimerization of STAT3 to study the role of p-STAT3 in IL-12-induced osteogenesis in BM-MSCs. Coincide with the results of interfering of IL-12Rß1 and TYK2, the promotion of activations of p-STAT3 and expressions of Runx2, Ogn in IL-12 co-cultured cells were also significant reduced by using CPT (Fig. 5a, b). Further more, the osteogenesis enhancement in IL-12co-cultured cells also eliminated in CPT treated cells (Fig. 5c, d). These results indicating the inhibition of STAT3 directly impaired the IL-12-induced osteogenesisin BM-MSCs.

IL-12 induced osteogenesis facilitated the bone famation and the irradiation hematopoiesis recovery

Basing on the better hematopoiesis recovery effect of IL-12 in irradiated mice was obtained when IL-12 was administrated within 24 hours after irradiation[21], we further estimated the bone formation and corresponding bone marrow hematopoiesis in femur of irradiated mice transplanted with BM-MSCs which were co-cultured with IL-12 (IL-12-BM-MSCs) for only 24 hours. For this, we transplantated IL-12BM-MSCs ,co-cultured with IL-12, into left femur, and transplantated BM-MSCs, co-cultured without IL-12, into right femur of a same individual irradiated mouse to exclude individual differences and to investigate the direct effect of IL-12-BM-MSCs on bone marrow hematopoiesis by minimally invasive surgery (supplementary Fig. 2a). As showed in Fig. 6a and b, femurs transplanted with IL-12-BM-MSCs showed increased cancellous bone mass than those transplanted with BM-MSCs. Parallelly to that, HSCPs in femurs transplanted with IL-12-BM-MSCs also had the more colony-formations (Fig. 6c, supplementary Fig. 2b).

Now, we confirmed that IL-12 could promote irradiation hematopoiesis recovery in vivo and confirmed its the osteogenic differentiations promotion in irradiated BM-MScs. For the latter, we are sure that IL-12Rß1 and its related signals, TYK2 and STAT3, played an important role, at least during the early period after irradiation. For that, we proposed the possible mechanism of IL-12 induced osteogenic differentiation in 
BM-MSCs after irradiation. Firstly, irradiation induced the swift upregulation of IL-12 receptors, especially IL-12Rß1. At this time, IL-12 triggered the downstream TYK2/STAT3 signaling and increased the STAT3 phosphorylation and then increased its dimerization and nuclear translocation. Then and lastly, the osteogenic genes expressions were promoted and the osteogenesis of BM-MSCs were enhanced and at the same time the hematopoiesis recovery happened (Fig. 7).

\section{Discussion}

As a multifunctional cytokine, IL-12 bioactivities involved in proliferation, differentiation, survival and apoptosis of various cell types $[19,26,27]$. What appeals to us is the considerate hematopoietic and gastrointestinal tract preventions and recovery functions in acute irradiation-induced of IL-12, which make it a brilliant promise application in irradiation protection[28, 29]. Even IL-12 was showed a unique ability to directly act on HSPCs to promote their proliferation and differentiation[30], in fact, considering the irradiation sensitivity of HSPCs, the function of HSPCs were seriously damaged in acute irradiation, it seemed hardly to explain the powerful hematopoiesis recovery of IL-12 only through its direct action on HSPCs. Considering the importance supportive role in the HM [31,32], the relatively more resistance to irradiation[33, 34], and osteoblasts differentiation potential of BM-MSCs which further directly responsible for bone formation, we natually suspected IL-12 may have effects on the osteogenesis of BM-MSCs to execute its hematopoietic supporting.

Previous researches indicated the powerful irradiation hematopoiesis recovery of IL-12 were obtained when it was used within 1 hour post irradiation or 24 hours before irradiation[21]. Therefore, here, all IL12administration of for animals or cells were conducted within 1 hour post irradiation unless otherwise specified. As expected, IL-12 did exert considerable hematopoiesis facilitation both in bone marrow and peripheral blood cells in irradiated mice during 21 days after irradiation. IL-12 also exhibited an apparent osteogenesis promotion both in vivo and in vitro ( see Fig. 2 ). Beside that, the significant better colonyformation abilities were also observed in IL-12 cured irradiated mice. All these results suggested IL-12 might really had a positive role in maintaining the function of $\mathrm{HM}$, such as maintaining osteogenic differentiation function of BM-MSCs, in irradiation.

As we known, IL-12 manifests its bioactivities through its receptors and their corresponding tyrosine kinases activate signaling cascade. The subsequent tyrosine phosphorylation of both the cytoplasmic proteins TYK2, interacting with IL-12Rß1, and JAK2, interacting with IL-12Rß2, and the subsequent activations of STAT4 and STAT3 are responsible for IL-12 signaling transduction[26, 27, 35]. Generally, the IL-12Rß2/ JAK2/STAT4 pathway and its activated IFN-gama play a major role in common bioactivities of IL-12, while IL-12Rß1 was appeared be not found direct function except indispensable subunit responsible for binding IL-12[36]. But more and more researches indicated that IL-12R 31 ,in single or in aiding IL-12 signalling, vary with cells-type, has many unknown functions [37]. Furthermore, the STAT3, one of the positive moleculars in IL-12 biological signal, was proved a pivotal role in maintaining host homeostasis, anti-tumors and immunomodulation, and the TYK2/STAT3 is constantly identified strong relationship with osteoblast differentiation and bone formation and can be activated by various 
cytokines, growth factors and other stimuli[38, 39]. Many osteogenic related factors, ALP, BMP2 and RUNX2, were also strongly associated with the actication of STAT3[27, 40]. Considering the rapid upregulating expression of IL-12R 31 in irradiated BM-MSCs, it seems that the more coincidence of IL12Rß1 expression peak, detected at hour 24 post irradiation, with the better IL-12-induced osteogenic effects, the more expression increasing of osteogenic genes and the more calcium nodule formation of cells co-cultured with IL-12 within one hours after irradiation, it is reasonable to speculate that the IL12Rß1/TYK2/STAT3 may have a crucial role in mediated IL-12-induced osteogenesis.

Therefore, next, we focused on the relationship of STAT3 activation and IL-12-induced osteogenic promotion. We estimated the activations of STAT3 in IL-12-BM-MSCs by interfering the possible upstream, IL-12R 1 and TYK2 signals, and directly inhibited its phosphorylation at tyrosine 705 by using special inhibitor (CPT). Interesting and predictable, BM-MSCs co-cultured with IL-12 showed an obvious activation of STAT3, and interfering IL-12Rß1 and TYK2 signal obviously abrogated the phosphorylations of STAT3 and upregulation of osteogenic genes. IL-12-induced osteogenesis enhancement appeared directly related to phosphorylation of tyrosine 705 of STAT3 in BM-MSCs, because there was significant ontogenesis eliminated in CPT treated cells. It worth noting that a short time, 24 hours, of co-culturing with IL-12 were used in all the interfering and inhibiting experiments. These finds not only suggested a crucial time-frame of IL-12-induced osteogenic promotion in irradiated BM-MSCs, but also confirmed a direct positive regulating relationship between IL-12R 31 , TYK2, p-STAT3 and osteogenic genes in IL-12induced osteogenic promotion in BM-MSCs.

At last, we parallelly examined the hematopoietic supportive of BM-MSCs co-cultured with IL-12 for 24 hours (IL-12-BM-MSCs) by HSPCs colony-forming assays, classical assays of the hematopoietic functions of bone marrow, and the bone formation of IL-12-BM-MSCs transplanted irradiated mice by analyzing the bone mineral density of the femur. Results showed an apparent more colonies and bone formations in IL-12-BM-MSCs transplanted mice. As we known that good bone formation is directly beneficial to reduce the bone loss which was common in irradiation[1] and is indirectly beneficial to reconstruction of hematopoiesis. These finds, even further researches are needed, suggested a the parallel relationship of the hematopoietic supportive and bone formative promotion of IL-12-BM-MSCs and further strengthened our suppose that IL-12 exert its hematopoietic supporting might though its bone mass formation enhancement functions on BM-MSCs.

\section{Conclusions}

The effects of IL-12 on osteogenesis promotion on BM-MSCs made it practical significance for researching its hematopoiesis recovery effects and the biological functions of BM-MSCs in bone marrow, and both of them are far from clarifying. It is tempting to clarify the different function and signal transduction of IL-12 in BM-MSCs for few studies demonstrated IL-12 participated in interfering osteoclasts formation alone and/or in association with the other cytokines [41-43]. Even further researches are needed, present study firstly showed IL-12 exerted a direct enhancement on osteogenic differentiation of irradiated BM-MSCs and its IL-12Rß1 signal pathway play an important role in that 
process. These results provide a new perspective regarding the protective role of IL-12 in hematopoiesis recovery after irradiation damage, and might help to develop new treatment strategy of irradiationinduced osteoporosis.

\section{Abbreviations}

BM-MSCs:bone marrow mesenchymal stem cells RT-qPCR:Real-time quantitative real-time PCR IL12:interleukin 12 WB:western blotting IL-12Rß1:IL-12 receptor beta 1 IL-12Rß2:IL-12 receptor beta 2 Runx2:runt-related transcription protein 2 OGN:osteoglycin TYK2:tyrosine-protein kinase 2 STAT3:Signal transducers and activators of transcription 3 JAK:Janus tyrosine kinase PBS:Phosphate-buffered saline GAPDH:glyceraldehyde-3-phosphate dehydrogenase FBS:foetal bovine serum RT:at room temperature DAPI:4,6-diamidino-2-phenylindole HSPCs:hematopoietic stem and progenitor cells

\section{Declarations}

\section{Ethical Approval and Consent to participate}

The experimental protocols were approved by the Animal Experiment Ethics Committee of Army Medical University institute. Consent was not applicable for this study.

No Human samples were used in this study.

\section{Consent for publication}

Not applicable.

\section{Availability of data and materials}

All data generated or analyzed during this study are included in this article [and its supplementary information files]. The materials described in our manuscript will be freely available for non-commercial purposes.

\section{Competing interests}

The authors declare that they have no competing interests.

\section{Fundings}

The authors extend their appreciation to the Chongqing Municipal Science and Technology Commission for funding this work through the People's Livelihood Science and technology innovation Foundation (No. CSTC 2015SHMSZX120078), the Achievement Transformation Fund of The Third Military Medical University (No. 2016D424) and the National Major Science and Technology Projects ( No. 2018ZX09J18103-004) 


\section{Authors' contributions}

Fengjie Li and Rong Zhang: Concepts, designing of the work, data analysis, data interpretation, manuscript preparation, editing and review; Changpeng Hu and Yang Yang performed parts of the data acquisition and analyses for cell culture. Qian ran and Yang Xiang conducted parts of experiments and prepared parts of the graphs and figures. Li Chen and Lixin Xiang conducted the immunofluorescence experiments. Gang Zhang and Zhongjun Li participated in the study design, experimental design and writing the manuscript. All authors read and approved the final manuscript.

\section{Acknowledgements}

Not applicable.

\section{Authors' information:}

Fengjie Li and Rong Zhang contributed equally to this work.

\section{Affiliations}

Department of Blood Transfusion, The Irradiation Biology Laboratory, The Second Affiliated Hospital, The Third Military Medical University, Chongqing, 400037, China

Department of Pharmacy, The Second Affiliated Hospital, The Third Military Medical University, Chongqing, 400037, China.

Department of Oral and Maxillofacial Surgery, The Second Affiliated Hospital, The Third Military Medical University, Chongqing, 400037, China

\section{Corresponding authors}

Correspondence to Gang Zhang or Zhongjun Li

\section{References}

1. Costa S, Reagan MR: Therapeutic Irradiation: Consequences for Bone and Bone Marrow Adipose Tissue. Front Endocrinol (Lausanne) 2019, 10:587.

2. Tohma $Y$, Dohi $Y$, Ohgushi $H$, Tadokoro $M$, Akahane $M$, Tanaka $Y$ : Osteogenic activity of bone marrowderived mesenchymal stem cells (BMSCs) seeded on irradiated allogenic bone. J Tissue Eng Regen Med 2012, 6:96-102.

3. Zhang X, Xiang L, Ran Q, Liu Y, Xiang Y, Xiao Y, Chen L, Li F, Zhong JF, Li Z: Crif1 Promotes Adipogenic Differentiation of Bone Marrow Mesenchymal Stem Cells After Irradiation by Modulating the PKA/CREB Signaling Pathway. Stem Cells 2015, 33:1915-1926. 
4. Cao X, Wu X, Frassica D, Yu B, Pang L, Xian L, Wan M, Lei W, Armour M, Tryggestad E, et al: Irradiation induces bone injury by damaging bone marrow microenvironment for stem cells. Proc Natl Acad Sci U S A 2011, 108:1609-1614.

5. Zhao K, Liu Q: The clinical application of mesenchymal stromal cells in hematopoietic stem cell transplantation. J Hematol Oncol 2016, 9:46.

6. Greenberger JS, Epperly M: Bone marrow-derived stem cells and radiation response. Semin Radiat Oncol 2009, 19:133-139.

7. Seshadri M, Qu CK: Microenvironmental regulation of hematopoietic stem cells and its implications in leukemogenesis. Curr Opin Hematol 2016, 23:339-345.

8. Moore KA: Recent advances in defining the hematopoietic stem cell niche. Curr Opin Hemato/2004, 11:107-111.

9. Zhu J, Emerson SG: A new bone to pick: osteoblasts and the haematopoietic stem-cell niche. Bioessays 2004, 26:595-599.

10. Calvi LM, Adams, G. B., Weibrecht, K. W., Weber, J. M., Olson, D. P., Knight, M. C., ... \& Milner, L. A.: Osteoblastic cells regulate the haematopoietic stem cell niche. Nature 2003, 425:836-841.

11. Zhang J, Niu C, Ye L, Huang H, He X, Tong WG, Ross J, Haug J, Johnson T, Feng JQ, et al: Identification of the haematopoietic stem cell niche and control of the niche size. Nature 2003, 425:836-841.

12. Muguruma Y, Yahata T, Miyatake H, Sato T, Uno T, Itoh J, Kato S, Ito M, Hotta T, Ando K: Reconstitution of the functional human hematopoietic microenvironment derived from human mesenchymal stem cells in the murine bone marrow compartment. Blood 2006, 107:1878-1887.

13. Sugrue T, Lowndes NF, Ceredig R: Mesenchymal stromal cells: radio-resistant members of the bone marrow. Immunol Cell Biol 2013, 91:5-11.

14. Wang Y, Zhu G, Wang J, Chen J: Irradiation alters the differentiation potential of bone marrow mesenchymal stem cells. Mol Med Rep 2016, 13:213-223.

15. Colombo MP, Trinchieri G: Interleukin-12 in anti-tumor immunity and immunotherapy. Cytokine Growth Factor Rev 2002, 13:155-168.

16. Kobayashi M, Fitz L, Ryan M, Hewick RM, Clark SC, Chan S, Loudon R, Sherman F, Perussia B, Trinchieri G: Identification and purification of natural killer cell stimulatory factor (NKSF), a cytokine with multiple biologic effects on human lymphocytes. J Exp Med 1989, 170:827-845.

17. Darlak KA, Wang Y, Li JM, Harris WA, Giver CR, Huang C, Waller EK: Host bone marrow-derived IL-12 enhances donor $\mathrm{T}$ cell engraftment in a mouse model of bone marrow transplantation. $J$ Hematol Oncol 2014, 7:16.

18. Chen T, Burke KA, Zhan Y, Wang X, Shibata D, Zhao Y: IL-12 facilitates both the recovery of endogenous hematopoiesis and the engraftment of stem cells after ionizing radiation. Exp Hematol 2007, 35:203-213. 
19. Gerber SA, Cummings RJ, Judge JL, Barlow ML, Nanduri J, Johnson DE, Palis J, Pentland AP, Lord EM, Ryan JL: Interleukin-12 preserves the cutaneous physical and immunological barrier after radiation exposure. Radiat Res 2015, 183:72-81.

20. Gokhale MS, Vainstein V, Tom J, Thomas S, Lawrence CE, Gluzman-Poltorak Z, Siebers N, Basile LA: Single low-dose rHulL-12 safely triggers multilineage hematopoietic and immune-mediated effects. Exp Hematol Oncol 2014, 3:11.

21. Basile LA, Ellefson D, Gluzman-Poltorak Z, Junes-Gill K, Mar V, Mendonca S, Miller JD, Tom J, Trinh A, Gallaher TK: HemaMax, a recombinant human interleukin-12, is a potent mitigator of acute radiation injury in mice and non-human primates. PLoS One 2012, 7:e30434.

22. Gluzman-Poltorak Z, Vainstein V, Basile LA: Association of Hematological Nadirs and Survival in a Nonhuman Primate Model of Hematopoietic Syndrome of Acute Radiation Syndrome. Radiat Res 2015, 184:226-230.

23. Gluzman-Poltorak Z, Mendonca SR, Vainstein V, Kha H, Basile LA: Randomized comparison of single dose of recombinant human IL-12 versus placebo for restoration of hematopoiesis and improved survival in rhesus monkeys exposed to lethal radiation. $J$ Hematol Oncol 2014, 7:31.

24. Klein RF: Osteoblasts make for good neighbors. Blood 2004, 103:3247-3247.

25. Calvi LM: Osteoblastic activation in the hematopoietic stem cell niche. Ann N Y Acad Sci 2006, 1068:477-488.

26. Watford WT, Hissong BD, Bream JH, Kanno Y, Muul L, O'Shea JJ: Signaling by IL-12 and IL-23 and the immunoregulatory roles of STAT4. Immunological Reviews 2004, 202:18.

27. Nicolaidou V, Wong MM, Redpath AN, Ersek A, Baban DF, Williams LM, Cope AP, Horwood NJ: Monocytes induce STAT3 activation in human mesenchymal stem cells to promote osteoblast formation. PLoS One 2012, 7:e39871.

28. Kerkar SP, Restifo NP: The power and pitfalls of IL-12. Blood 2012, 119:4096-4097.

29. Singh VK, Romaine PL, Newman VL: Biologics as countermeasures for acute radiation syndrome: where are we now? Expert Opin Biol Ther 2015, 15:465-471.

30. Chiba Y, Mizoguchi I, Hasegawa H, Ohashi M, Orii N, Nagai T, Sugahara M, Miyamoto Y, Xu M, Owaki T, Yoshimoto T: Regulation of myelopoiesis by proinflammatory cytokines in infectious diseases. Cell Mol Life Sci 2018, 75:1363-1376.

31. Abbuehl JP, Tatarova Z, Held W, Huelsken J: Long-Term Engraftment of Primary Bone Marrow Stromal Cells Repairs Niche Damage and Improves Hematopoietic Stem Cell Transplantation. Cell Stem Cell 2017, 21:241-255 e246.

32. Wu J, Zhang W, Ran Q, Xiang Y, Zhong JF, Li SC, Li Z: The Differentiation Balance of Bone Marrow Mesenchymal Stem Cells Is Crucial to Hematopoiesis. Stem Cells Int 2018, 2018:1540148.

33. Chen MF, Lin CT, Chen WC, Yang CT, Chen CC, Liao SK, Liu JM, Lu CH, Lee KD: The sensitivity of human mesenchymal stem cells to ionizing radiation. Int J Radiat Oncol Biol Phys 2006, 66:244-253. 
34. Singh S, Kloss FR, Brunauer R, Schimke M, Jamnig A, Greiderer-Kleinlercher B, Klima G, Rentenberger $J$, Auberger T, Hachl O, et al: Mesenchymal stem cells show radioresistance in vivo. J Cell Mol Med 2012, 16:877-887.

35. Feng X, Shen S, Cao P, Zhu L, Zhang Y, Zheng K, Feng G, Zhang D: The role of oncostatin M regulates osteoblastic differentiation of dental pulp stem cells through STAT3 pathway. Cytotechnology 2016, 68:2699-2709.

36. Weaver JR, Nadler JL, Taylor-Fishwick DA: Interleukin-12 (IL-12)/STAT4 Axis Is an Important Element for beta-Cell Dysfunction Induced by Inflammatory Cytokines. PLoS One 2015, 10:e0142735.

37. Robinson RT: IL12Rbeta1: the cytokine receptor that we used to know. Cytokine 2015, 71:348-359.

38. Fan D, Chen Z, Chen Y, Shang Y: Mechanistic roles of leptin in osteogenic stimulation in thoracic ligament flavum cells. J Biol Chem 2007, 282:29958-29966.

39. Derecka M, Gornicka A, Koralov SB, Szczepanek K, Morgan M, Raje V, Sisler J, Zhang Q, Otero D, Cichy J, et al: Tyk2 and Stat3 regulate brown adipose tissue differentiation and obesity. Cell Metab 2012, 16:814-824.

40. Itoh S, Udagawa N, Takahashi N, Yoshitake F, Narita H, Ebisu S, Ishihara K: A critical role for interleukin- 6 family-mediated Stat3 activation in osteoblast differentiation and bone formation. Bone 2006, 39:505-512.

41. Horwood NJ, Elliott J, Martin TJ, Gillespie MT: IL-12 Alone and in Synergy with IL-18 Inhibits Osteoclast Formation In Vitro. The Journal of Immunology 2001, 166:4915-4921.

42. Kitaura H, Fujimura Y, Yoshimatsu M, Kohara H, Morita Y, Aonuma T, Fukumoto E, Masuyama R, Yoshida N, Takano-Yamamoto T: IL-12- and IL-18-mediated, nitric oxide-induced apoptosis in TNFalpha-mediated osteoclastogenesis of bone marrow cells. Calcif Tissue Int 2011, 89:65-73.

43. Yoshimatsu M, Kitaura H, Fujimura Y, Kohara H, Morita Y, Yoshida N: IL-12 Inhibits Lipopolysaccharide Stimulated Osteoclastogenesis in Mice. J Immunol Res 2015, 2015:214878.

\section{Figures}


a

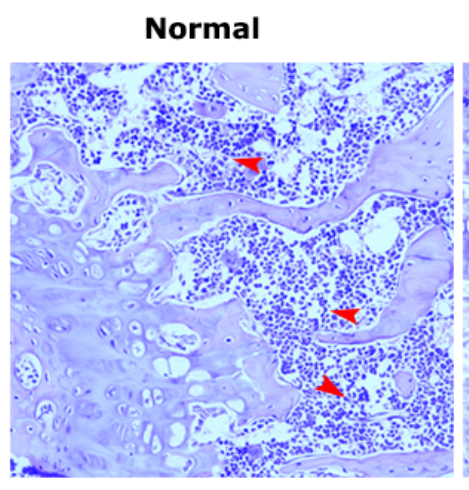

IR

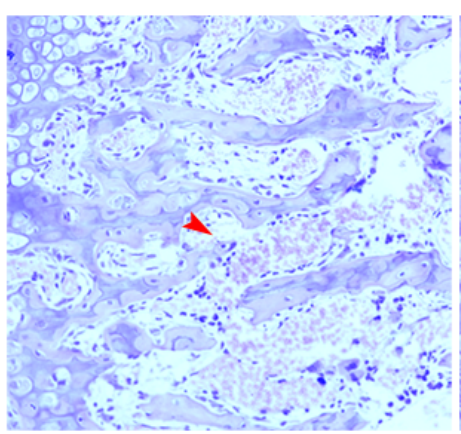

IR+IL-12

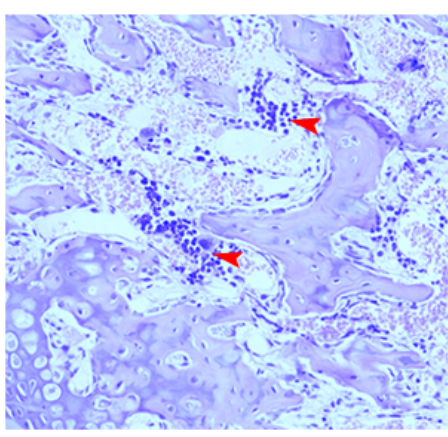

b

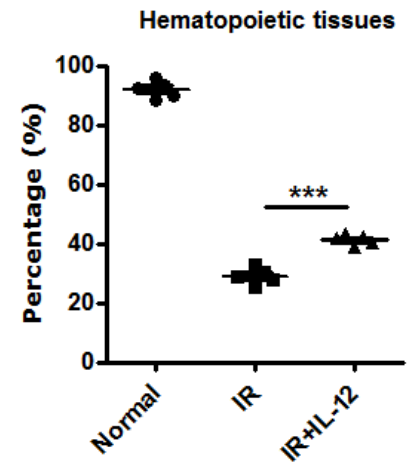

C
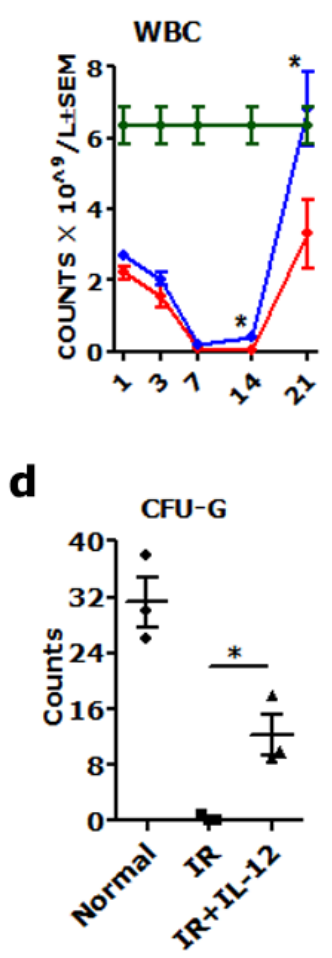
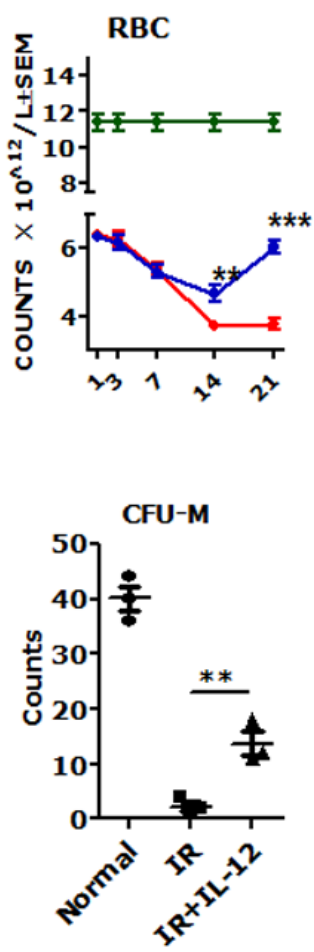
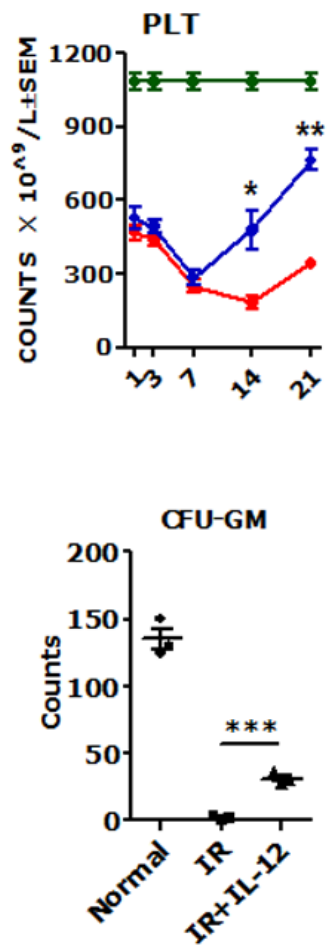
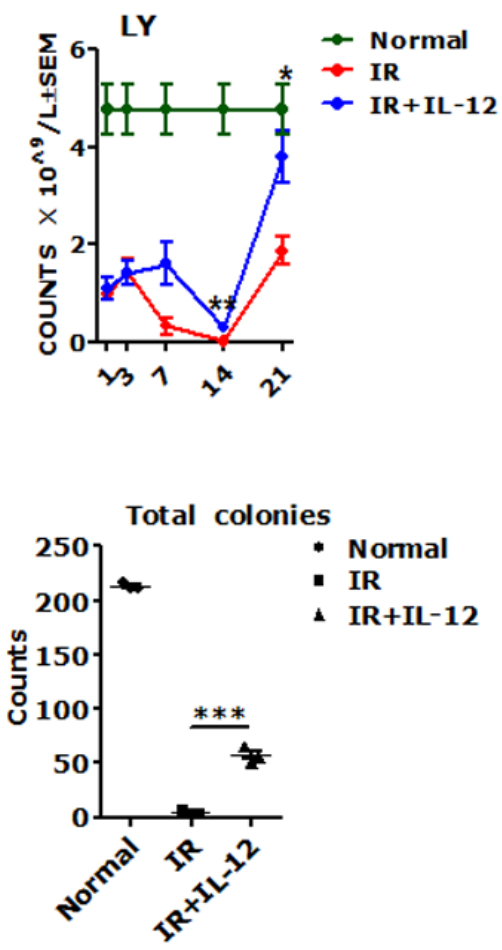

\section{Figure 1}

IL-12 promoted the hematopoiesis recovery in 5 Gy irradiated mice. (a) HE staining of femurs of irradiated and subcutaneous injected with a single dose of $1 \mathrm{ng} / \mathrm{g} \mathrm{IL-12} \mathrm{mice.} \mathrm{The} \mathrm{magnifications} \mathrm{are} \mathrm{200x.}$

Hemetopoiesis tissues were showed at the arrows. (b) The percentage of hematopoietic tissues of the femoral bone tissue sections of irradiated and injected with a single dose of $1 \mathrm{ng} / \mathrm{g} \mathrm{IL-12} \mathrm{mice.} \mathrm{(c)} \mathrm{The}$ peripheral blood cells counts and the HSPCs colonies of irradiated and injected with a single dose of 1 $\mathrm{ng} / \mathrm{g} \mathrm{IL-12} \mathrm{mice}$ 

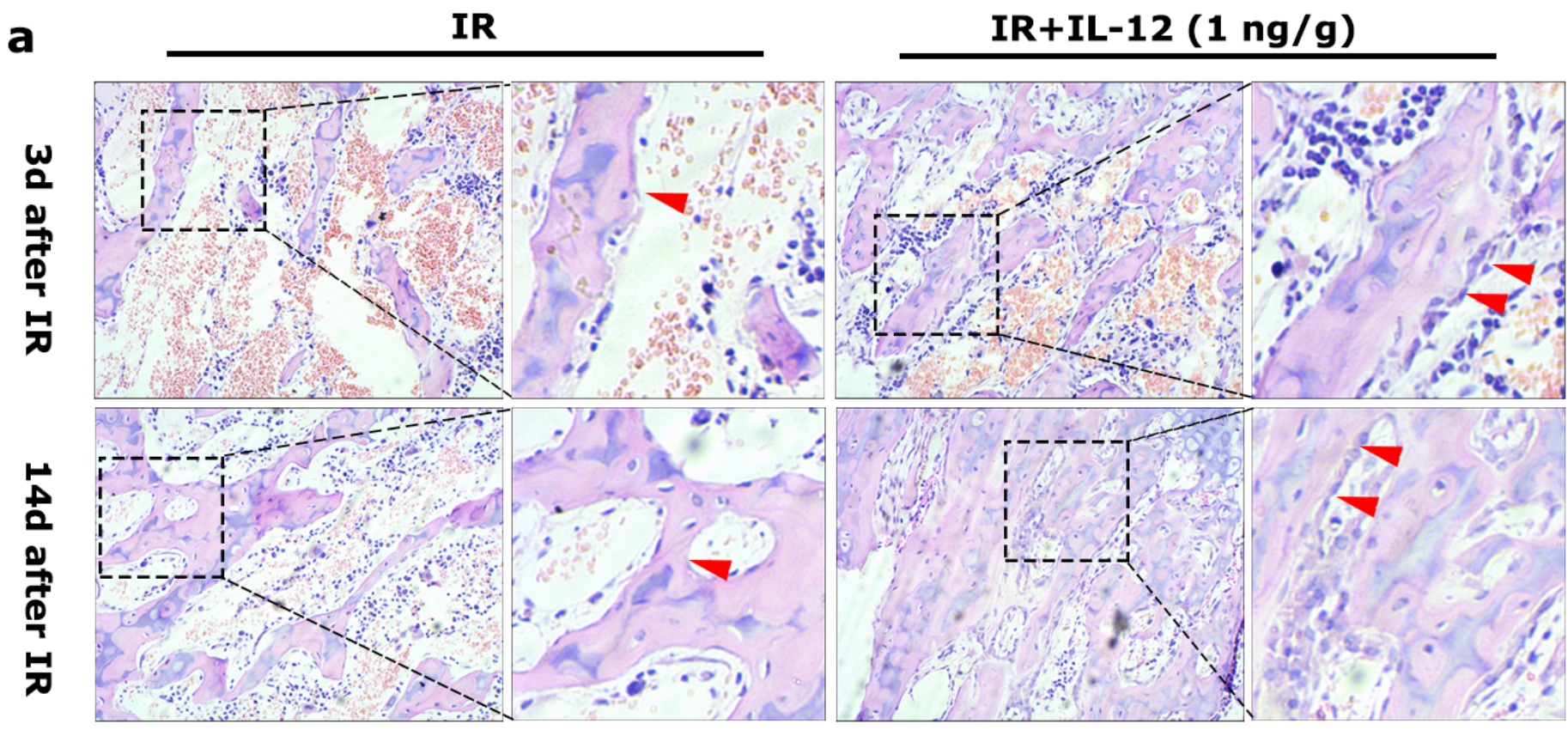

b

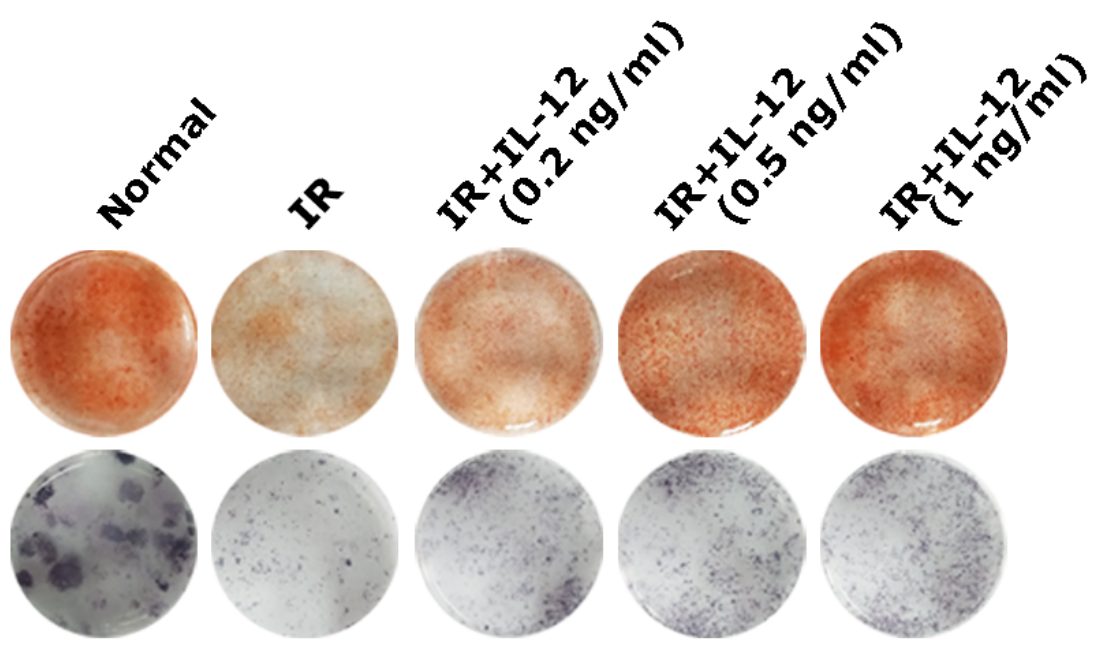

C

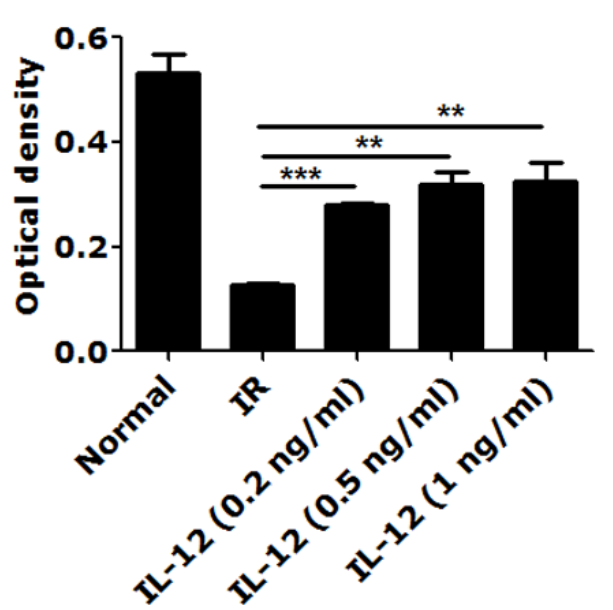

Figure 2

The osteogenesis effects of IL-12 in 5 Gy irradiated mice and 9 Gy irradiated BM-MSCs. (a) HE staining of femur sections and osteoblasts (at the arrow). (b) Alzarin red S staining of cells calcium depositions and the ALP activities staining of osteoblasts differentiated cells in irradiated BM-MSCs co-cultured with 0.2 , $0.5,1 \mathrm{ng} / \mathrm{ml} \mathrm{IL}-12$ in osteogenesis inducing medium for 14 days. The magnifications are $200 \times$. (c-d) Quantification of calcium depositions using assays of colorimetric determination of the dye extraction in cells stained with by alizarin red. The determination absorption wavelength was $562 \mathrm{~nm}$. All the data were presented as the mean $\pm S E M, n=3$. 
b
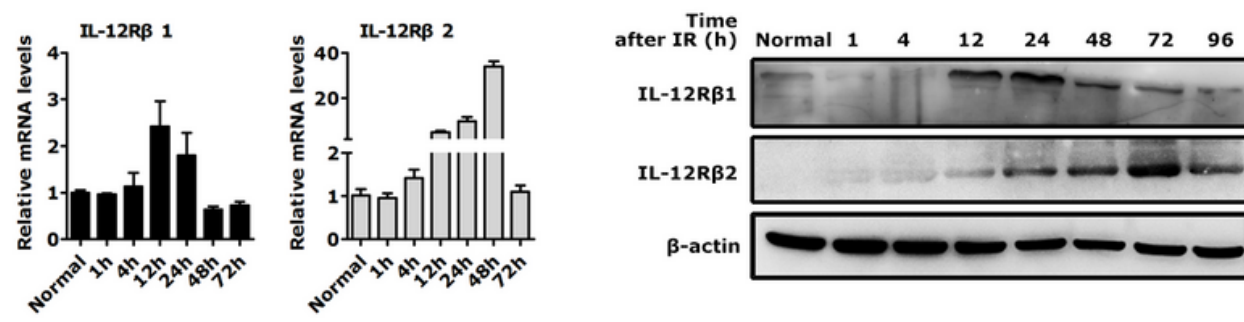

d

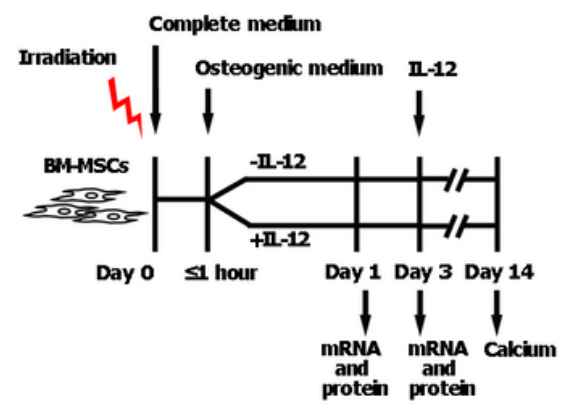

f

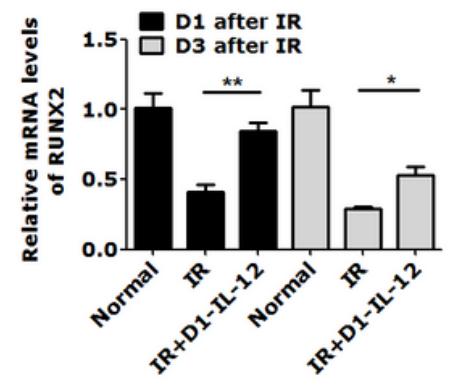

$\mathbf{g}$

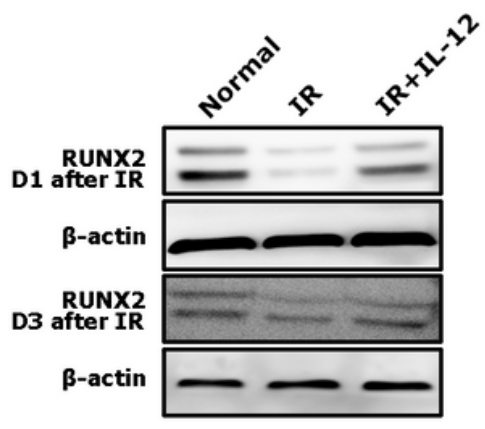

b

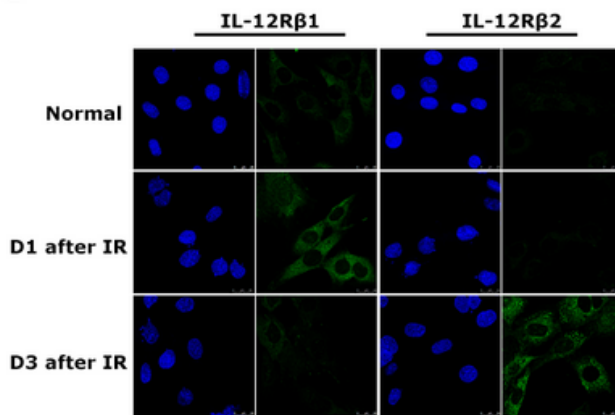

$\mathbf{e}$

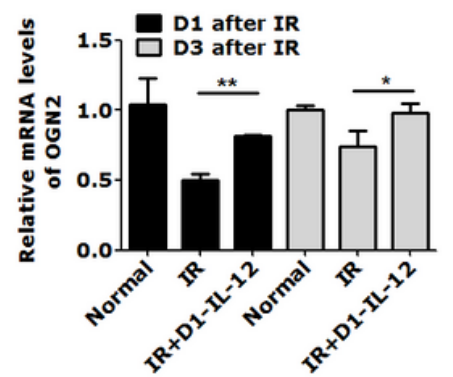

h

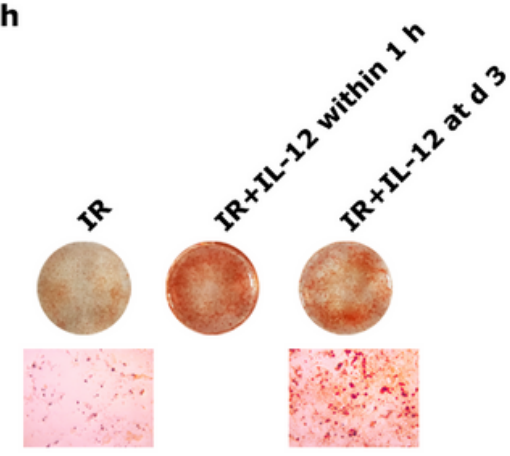

\section{Figure 3}

The expression of IL-12 receptors and the osteogenic process of BM-MSCs co-cultured with $0.2 \mathrm{ng} / \mathrm{ml} \mathrm{IL-}$ 12 in osteogenesis inducing medium after irradiation. (a-c) RT-PCR, Western blot and immunofluoresence analysis of expressions of IL-12Rß1 and IL-12Rß2 (d-g) The mRNA and protein levels of Runx2 and Ogn in irradiated cells co-cultured with IL-12 in osteogenesis inducing medium for 1 and 3 days. Three replicates were used in the mRNA levels analysis and data were presented as the mean \pm SEM. (h) Alzarin red S staining of calcium depositions in cells co-cultured IL-12 on day 1 and day 3 after irradiation in osteogenesis inducing medium for 14 days. The magnifications are $200 \times$. 
a

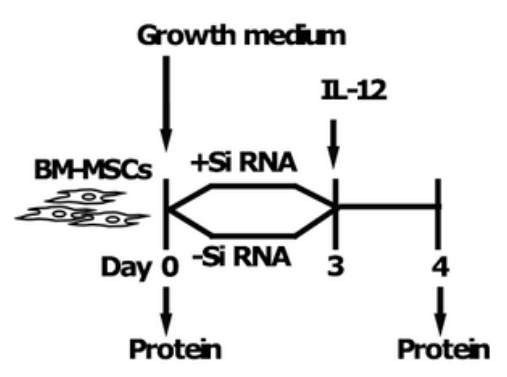

C

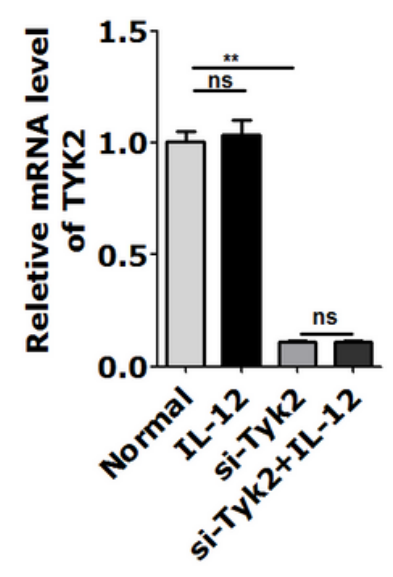

e
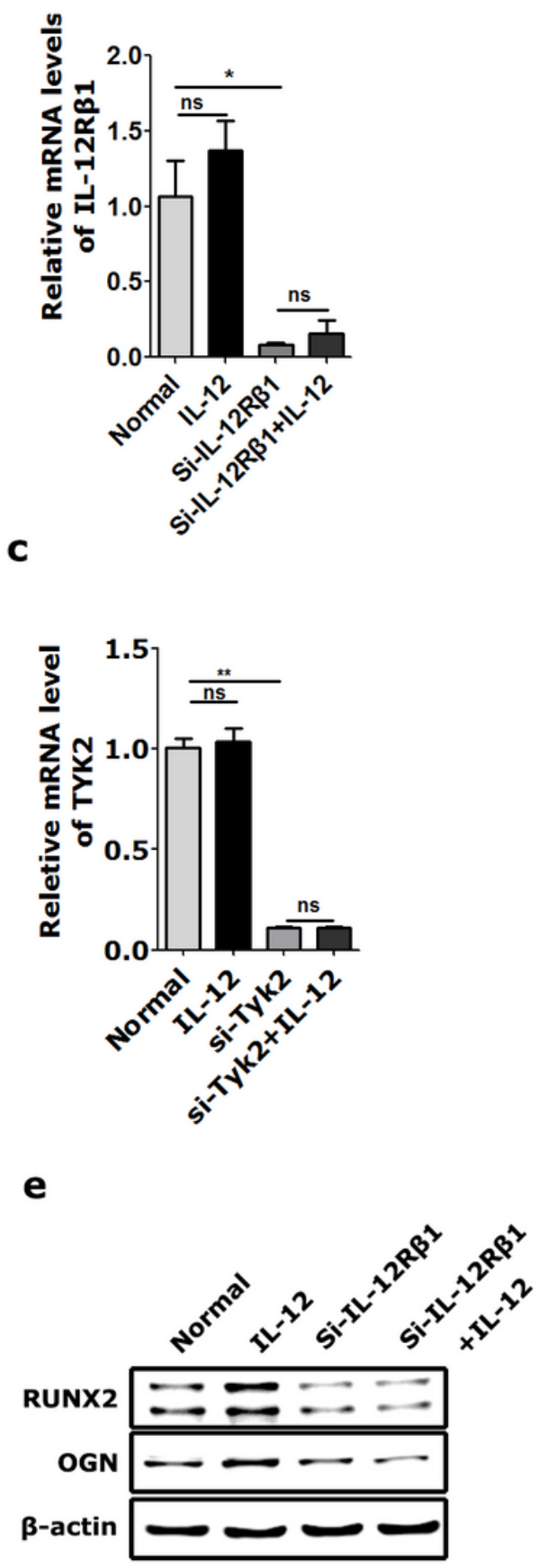

b

d
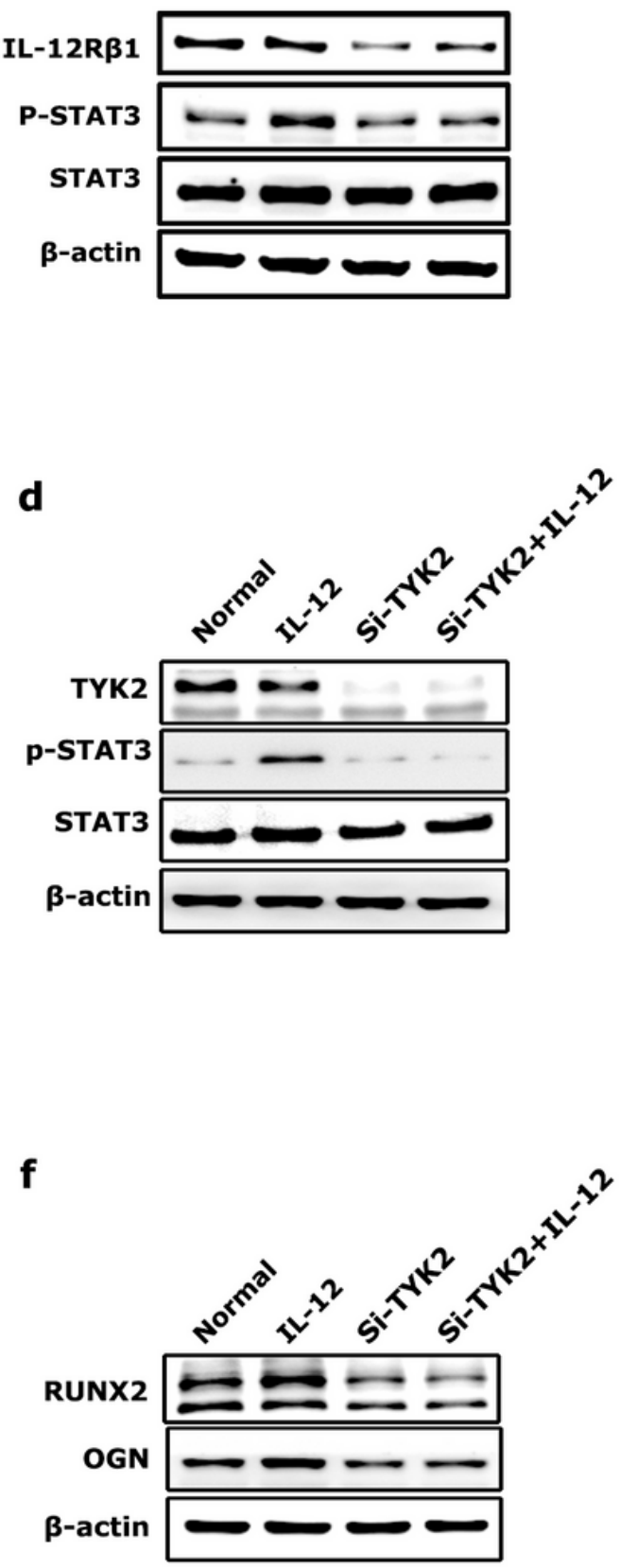

Figure 4

The role of IL-12Rß1/TYK2 signal in IL-12-induced osteogenesis of BM-MSCs. (a-d) qRT-PCR analysis and western blot analysis of expressions of IL-12Rß1, STAT3, and p-STAT3 in IL-12Rß1 and TYK2 siRNA transfected cells co-cultured in complete media with or without $0.2 \mathrm{ng} / \mathrm{ml} \mathrm{IL-12} \mathrm{for} 24$ hours. All the data were presented as the mean $\pm S E M, n=3$. (c) Western blot analysis of expressions of RUNX2, OGN in IL12Rß1 and TYK2 siRNA transfected co-cultured in osteogenesis inducing medium with or without 0.2 $\mathrm{ng} / \mathrm{ml} \mathrm{IL-12} \mathrm{for} 24$ hours. The cells without transfection cultured without IL-12 used as the controls. 
a
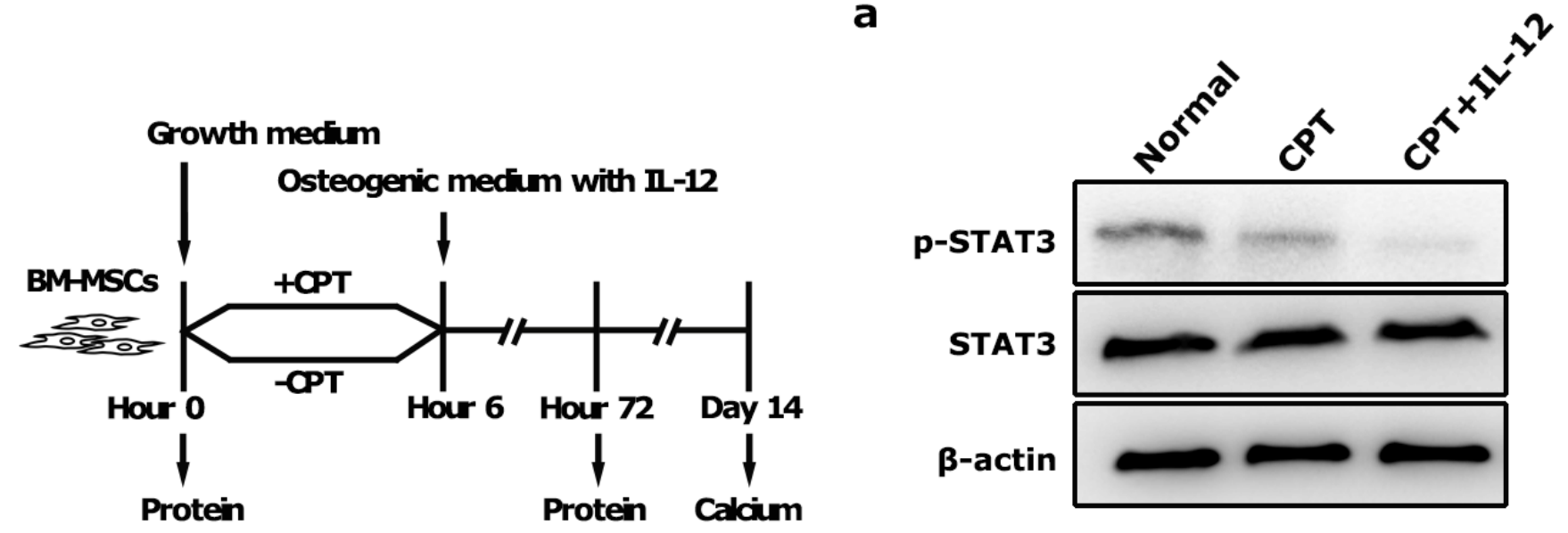

b

C

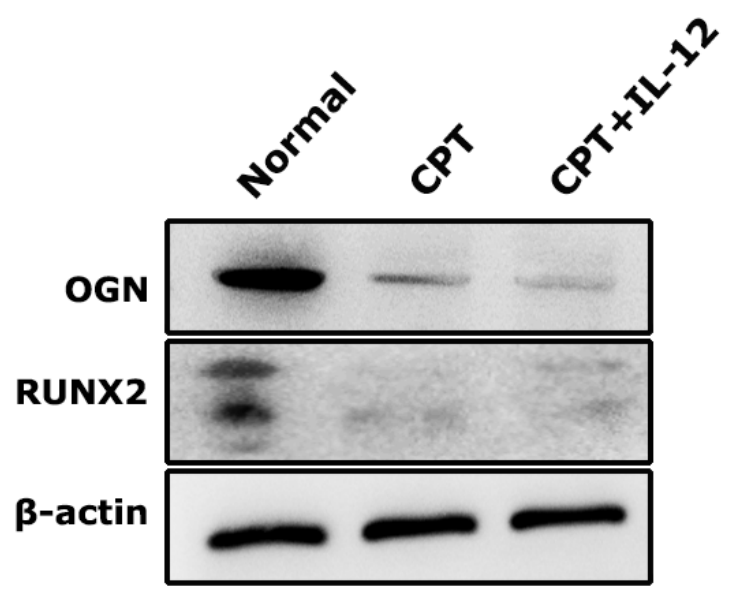

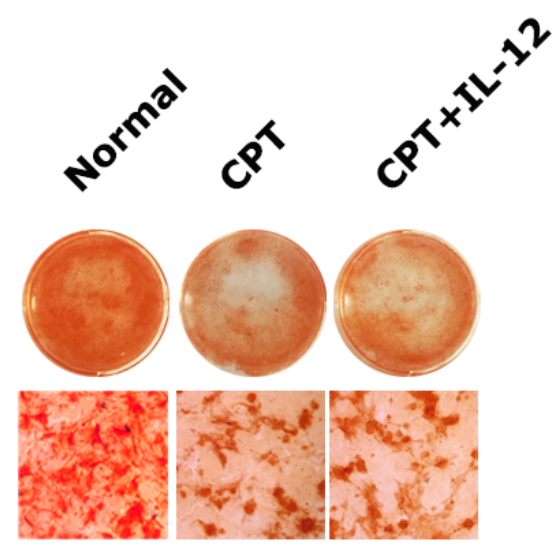

d

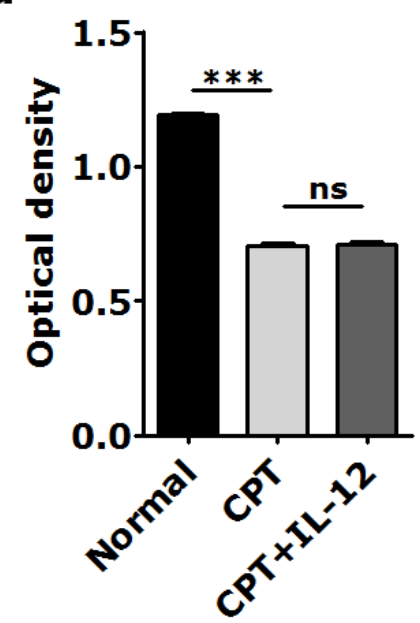

Figure 5

The role of STAT3 activation in IL-12-induced osteogenesis of BM-MSCs. (a) Western blot analysis of STAT3, p-STAT3 and TYK2 expressions in CPT treated cells co-cultured in osteogenesis inducing medium with or without $0.2 \mathrm{ng} / \mathrm{ml} \mathrm{IL-12} \mathrm{for} 24$ hours (b) Western blot analysis of RUNX2, OGN, TYK2 expressions in CPT treated cells co-cultured in osteogenesis inducing medium with or without $0.2 \mathrm{ng} / \mathrm{ml} \mathrm{IL-12} \mathrm{for} 24$ hours (c-d) Alzarin red S staining of calcium depositions, the magnifications are $200 x$, and quantification of calcium depositions using assay of colorimetric determination of the dye extraction in cells stained with by alizarin red. The determination absorption wavelength was $562 \mathrm{~nm}$. All the data were presented as the mean \pm SEM, $n=3$. 


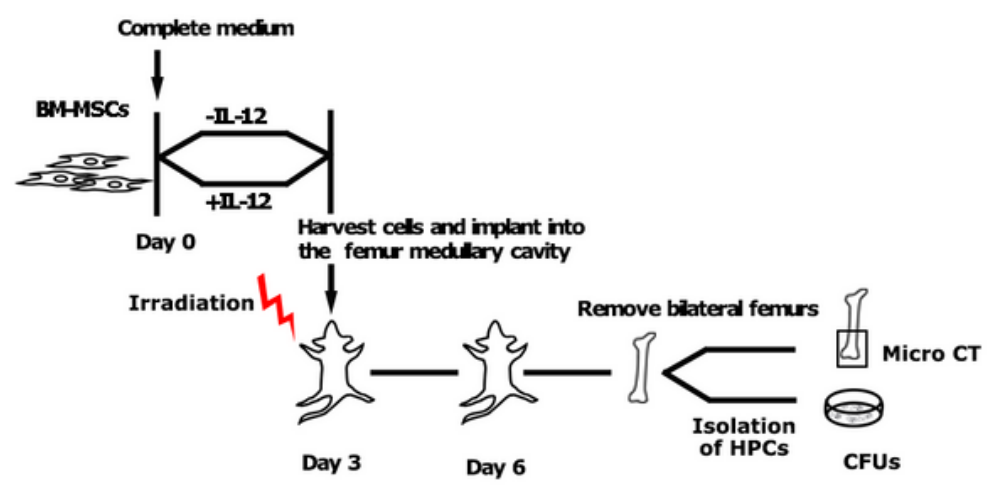

a

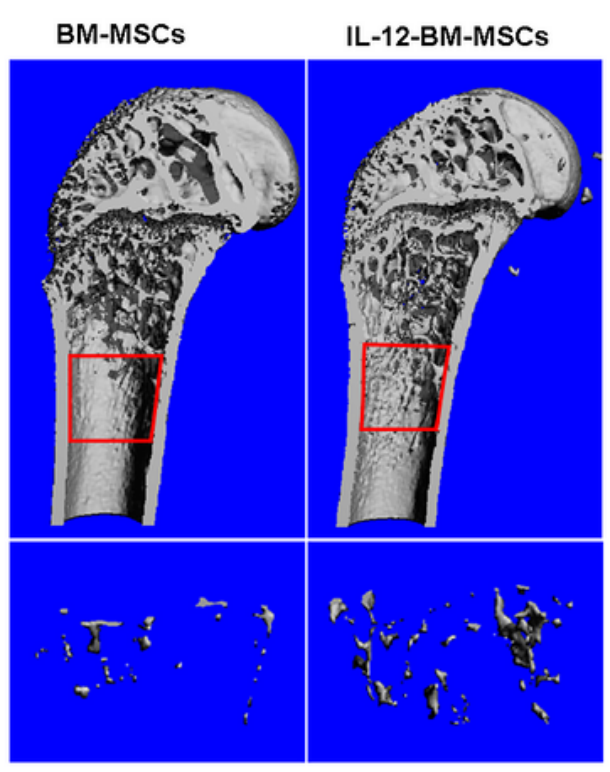

C

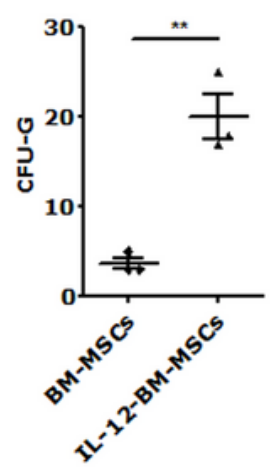

b
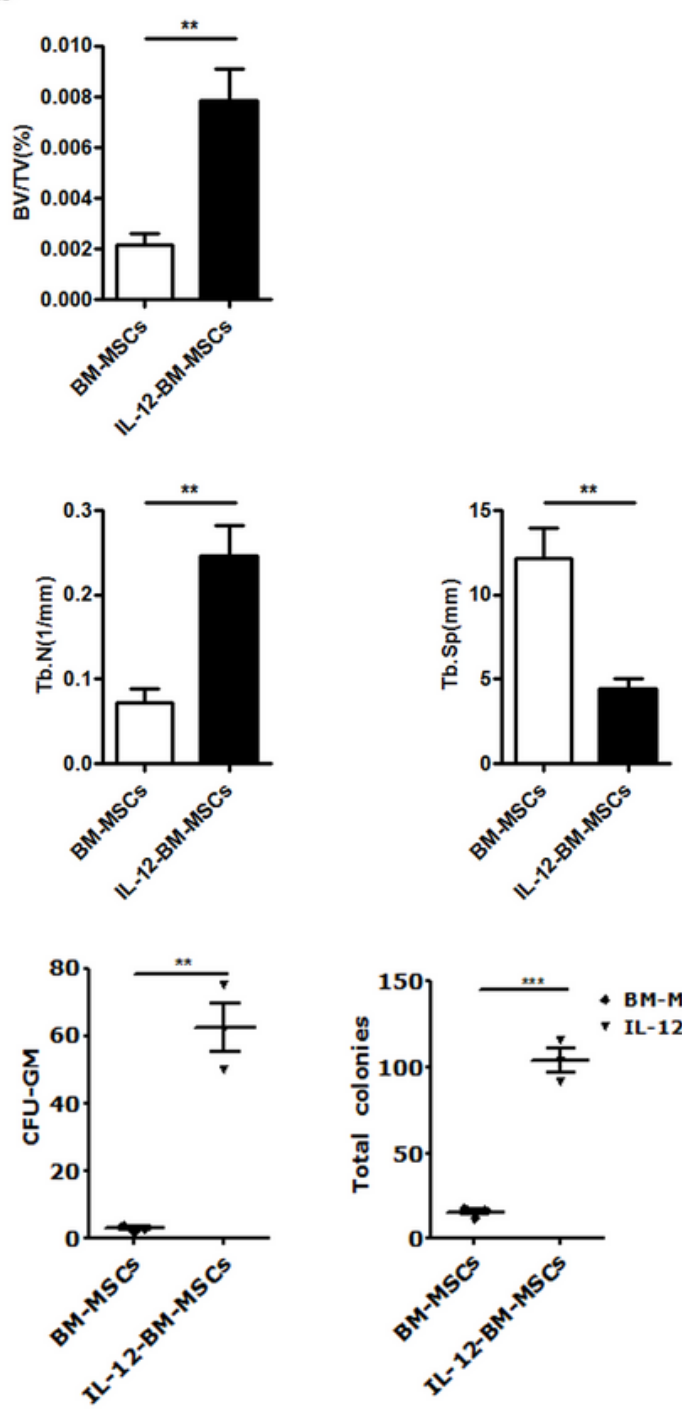

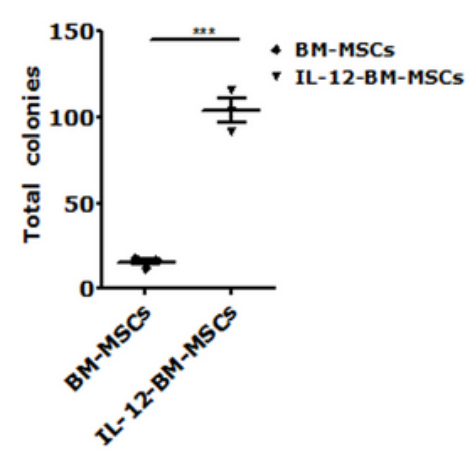

\section{Figure 6}

The hematopoiesis surporting and bone formations of BM-MSCs co-cultured with $0.2 \mathrm{ng} / \mathrm{ml} \mathrm{IL-12} \mathrm{for} 24$ hours . $(a, b)$ Bone mineral density analysis of bilatteral femur of $5 \mathrm{~Gy}$ irradiated mice which were cells transplantated at the bilatteral femur by using Scanco Medical CT-40 instruments. All the data were presented as the mean $\pm S E M, n=5$. (c) HSPCs colonies analysis bilatteral femur of 5 Gy irradiated mice which were cells transplantated at the bilatteral fem 


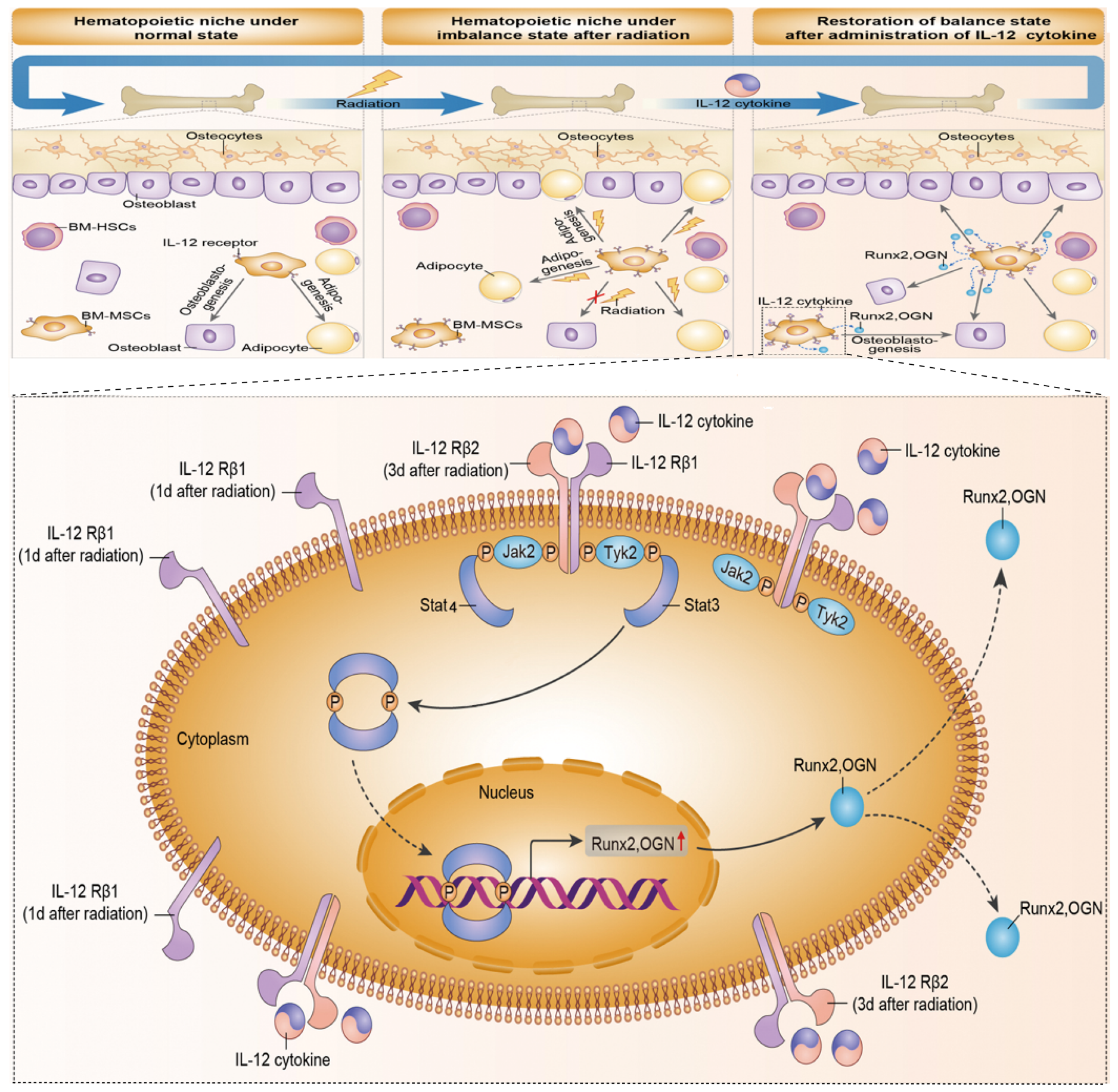

\section{Figure 7}

Proposed mechanism of IL-12-induced osteogenesis in BM-MSCs after irradiation. Firstly, irradiation induced the upregulation of IL-12 receptors, especially IL-12Rß1. Then the TYK2/STAT3 signaling activation, such as TYK2 activation, STAT3 phosphorylation, nuclear translocation after dimerization, were triggered. In succession, the expression initial of osteogenic genes, RUNX2 and OGN, were increased, which in turn promoted the osteoblast differentiation of BM-MSCs. $\uparrow=$ increase.

\section{Supplementary Files}


This is a list of supplementary files associated with this preprint. Click to download.

- Supplementfigure1.tif

- Supplementfigure2.tif 